Correction

\title{
Correction: Tang, C. Y. and Chen, X.Y. A Class of Coning Algorithms Based on a Half-Compressed Structure. Sensors 2014, 14, 14289-14301
}

\section{Chuanye Tang and Xiyuan Chen*}

Key Laboratory of Micro-Inertial Instrument and Advanced Navigation Technology, Ministry of Education, School of Instrument Science and Engineering, Southeast University, Sipailou 2, Nanjing 210096, China; E-Mail: 230129460@seu.edu.cn

* Author to whom correspondence should be addressed; E-Mail: chxiyuan@seu.edu.cn; Tel./Fax: +86-25-8379-2010.

Academic Editor: Vittorio M.N. Passaro

Received: 23 December 2014 / Accepted: 11 February 2015 / Published: 13 February 2015

\section{Change in Tables/Equations}

Due to an oversight by MDPI and the authors, the following numerical corrections were not made in the originally published article [1]. MDPI-Sensors and the authors would like to apologize for any inconvenience brought to the readers.

The authors wish to make the following correction to the article [1]:

The former Table 9 (labelled here as Old Table 9) and Table 10 (labelled here as Old Table 10) should be replaced by the new versions shown below (labelled here as New Table 9 and New Table 10), respectively. The $z \mathrm{~s}$ in Tables 15 and 16 and the maneuver errors in [1] Table 17 will not be affected by the correction to Tables 9 and 10, because these $z \mathrm{~s}$ and the maneuver errors were all calculated using the correct coefficients in New Tables 9 and 10. That means, the mistakes in Old Tables 9 and 10 are just writing errors.

Old Table 9. FTSuc algorithm coefficients.

\begin{tabular}{ccc}
\hline $\boldsymbol{L}$ & $\boldsymbol{N}$ & Coefficients \\
\hline 3 & 3 & $\varsigma_{12}=\varsigma_{23}=27 / 40, \varsigma_{13}=9 / 20$ \\
\hline 4 & 4 & $\varsigma_{12}=\varsigma_{34}=232 / 315, \varsigma_{23}=178 / 315, \varsigma_{13}=\varsigma_{24}=46 / 105, \varsigma_{14}=54 / 105$ \\
\hline \multirow{2}{*}{5} & \multirow{2}{*}{5} & $\varsigma_{12}=18575 / 24192, \varsigma_{13}=2675 / 6048, \varsigma_{14}=11,225 / 24,192, \varsigma_{15}=125 / 252, \varsigma_{23}=2575 / 6048$, \\
& $\varsigma_{24}=425 / 672, \varsigma_{25}=139,75 / 24,192, \varsigma_{34}=1975 / 3024, \varsigma_{35}=325 / 1512, \varsigma_{45}=21,325 / 24,192$ \\
\hline
\end{tabular}


New Table 9. FTSuc algorithm coefficients.

\begin{tabular}{ccc}
\hline $\boldsymbol{L}$ & $\boldsymbol{N}$ & Coefficients \\
\hline 3 & 3 & $\varsigma_{12}=\varsigma_{23}=27 / 40, \varsigma_{13}=9 / 20$ \\
\hline 4 & 4 & $\varsigma_{12}=\varsigma_{34}=232 / 315, \varsigma_{23}=178 / 315, \varsigma_{13}=\varsigma_{24}=46 / 105, \varsigma_{14}=54 / 105$ \\
\hline \multirow{2}{*}{5} & 5 & $\varsigma_{12}=21325 / 24192, \varsigma_{13}=325 / 1512, \varsigma_{14}=13975 / 24192, \varsigma_{15}=125 / 252, \varsigma_{23}=1975 / 3024$, \\
& $\varsigma_{24}=425 / 672, \varsigma_{25}=11225 / 24192, \varsigma_{34}=2575 / 6048, \varsigma_{35}=2675 / 6048, \varsigma_{45}=18575 / 24192$ \\
\hline
\end{tabular}

Old Table 10. LMSuc algorithm coefficients.

\begin{tabular}{ccc}
\hline $\boldsymbol{L}$ & $\boldsymbol{N}$ & Coefficients \\
\hline 3 & 3 & $\varsigma_{12}=0.681306, \varsigma_{13}=0.444312, \varsigma_{23}=0.679452$ \\
\hline 4 & 4 & $\varsigma_{12}=0.739716, \varsigma_{13}=0.432467, \varsigma_{14}=516734, \varsigma_{23}=0.571812, \varsigma_{24}=0.4434453, \varsigma_{34}=0.737795$ \\
\hline \multirow{2}{*}{5} & 5 & $\varsigma_{12}=769,240, \varsigma_{13}=0.438591, \varsigma_{14}=0.467191, \varsigma_{15}=0.495116, \varsigma_{23}=0.431753, \varsigma_{24}=0.625867$, \\
& $\varsigma_{25}=0.579681, \varsigma_{34}=0.656805, \varsigma_{35}=0.213527, \varsigma_{45}=0.881820$ \\
\hline
\end{tabular}

New Table 10. LMSuc algorithm coefficients.

\begin{tabular}{ccc}
\hline $\boldsymbol{L}$ & $\boldsymbol{N}$ & Coefficients \\
\hline 3 & 3 & $\varsigma_{12}=0.679452, \varsigma_{13}=0.444312, \varsigma_{23}=0.681306$ \\
\hline 4 & 4 & $\varsigma_{12}=0.737795, \varsigma_{13}=0.434453, \varsigma_{14}=516734, \varsigma_{23}=0.571812, \varsigma_{24}=0.432467, \varsigma_{34}=0.739716$ \\
\hline \multirow{2}{*}{5} & 5 & $\varsigma_{12}=0.881820, \varsigma_{13}=0.213527, \varsigma_{14}=0.579681, \varsigma_{15}=0.495116, \varsigma_{23}=0.656805, \varsigma_{24}=0.625867$, \\
& $\varsigma_{25}=0.467191, \varsigma_{34}=0.431753, \varsigma_{35}=0.438591, \varsigma_{45}=0.769240$
\end{tabular}

The former Equation (12) of [1]:

$$
\begin{array}{r}
G \Gamma\left(t_{l-1}\right)=\bar{G}, G \equiv\left(g_{i}\right)_{M \times 1}, \bar{G} \equiv\left(\bar{g}_{j}\right)_{M \times 1}, \Gamma\left(t_{l-1}\right) \equiv\left(\gamma_{j i}\left(t_{l-1}\right)\right)_{M \times M} \\
\gamma_{j i}\left(t_{l-1}\right)=\left\{\begin{array}{cc}
\left(-t_{l-1}\right)^{i-j} & , j=1 \\
\left(-t_{l-1}\right)^{i-j}(i-1) ! /(j-1) !, 1<j \leq i & , j>i
\end{array}\right.
\end{array}
$$

Should be replaced by the new Equation (12):

$$
\begin{gathered}
G \Gamma\left(t_{l-1}\right)=\bar{G}, G \equiv\left(g_{i}\right)_{M \times 1}, \bar{G} \equiv\left(\bar{g}_{j}\right)_{M \times 1}, \Gamma\left(t_{l-1}\right) \equiv\left(\gamma_{j i}\left(t_{l-1}\right)\right)_{M \times M} \\
\left(-t_{l-1}\right)^{i-j}, \quad j=1 \\
\gamma_{j i}\left(t_{l-1}\right)=\left\{\begin{array}{c}
\left(-t_{l-1}\right)^{i-j}(i-1) ! /((i-j) !(j-1) !), 1<j \leq i \\
0 \quad, j>i
\end{array}\right.
\end{gathered}
$$

Affected by the correction to Equation (12), the former Table 17 (labelled here as Old Table 17) of [1] should be replaced by the new version (labelled here as New Table 17). The correction to Table 17 will not affect the conclusions of [1]. 
Old Table 17. Maximum maneuver error over $2 \mathrm{~s}$ maneuver.

\begin{tabular}{cccccccc}
\hline \multirow{2}{*}{$\boldsymbol{L}$} & \multirow{2}{*}{$\boldsymbol{N}$} & \multicolumn{6}{c}{ Maximum Maneuver Error, $\boldsymbol{\mu}$ Rad } \\
\cline { 3 - 7 } & & FTSc & LMSc & FTShe & LMShc & FTSuc & LMSuc \\
\hline 3 & 3 & $1.00 \mathrm{e}-2$ & $-1.88 \mathrm{e}-2$ & $-3.34 \mathrm{e}-3$ & $3.65 \mathrm{e}-3$ & $2.86 \mathrm{e}-6$ & $-2.52 \mathrm{e}-2$ \\
4 & 4 & $3.24 \mathrm{e}-2$ & $3.25 \mathrm{e}-2$ & $-5.51 \mathrm{e}-3$ & $-5.54 \mathrm{e}-3$ & $1.48 \mathrm{e}-12$ & $9.66 \mathrm{e}-4$ \\
5 & 5 & $7.32 \mathrm{e}-2$ & $7.33 \mathrm{e}-2$ & $-7.50 \mathrm{e}-3$ & $-7.52 \mathrm{e}-3$ & $-7.23 \mathrm{e}-13$ & $3.25 \mathrm{e}-5$ \\
\hline
\end{tabular}

New Table 17. Maximum maneuver error over $2 \mathrm{~s}$ maneuver.

\begin{tabular}{cccccccc}
\hline \multirow{2}{*}{$\boldsymbol{L}$} & \multirow{2}{*}{$\boldsymbol{N}$} & \multicolumn{6}{c}{ Maximum Maneuver Error, $\boldsymbol{\mu}$ rad } \\
\cline { 3 - 7 } & & FTSc & LMSc & FTShe & LMShc & FTSuc & LMSuc \\
\hline 3 & 3 & $-1.09 \mathrm{e}-2$ & $-7.29 \mathrm{e}-3$ & $3.63 \mathrm{e}-3$ & $7.48 \mathrm{e}-3$ & $1.39 \mathrm{e}-6$ & $3.66 \mathrm{e}-3$ \\
4 & 4 & $-3.52 \mathrm{e}-2$ & $-3.54 \mathrm{e}-2$ & $5.98 \mathrm{e}-3$ & $5.89 \mathrm{e}-3$ & $1.67 \mathrm{e}-12$ & $-1.40 \mathrm{e}-4$ \\
5 & 5 & $-7.97 \mathrm{e}-2$ & $-7.97 \mathrm{e}-2$ & $8.15 \mathrm{e}-3$ & $8.17 \mathrm{e}-3$ & $-3.09 \mathrm{e}-13$ & $-4.73 \mathrm{e}-6$ \\
\hline
\end{tabular}

\section{Change in Main Body Paragraphs}

Due to an obscurity on how Equations (13) and (14) of [1] were built, the authors wish to insert some additional sentences to explain how Equations (13) and (14) of [1] can be converted from Equations (59) and (13) of Song (reference [9] of [1]).

Below we respectively denote the Song $\varsigma_{i j}$ and the $[1] \varsigma_{i j}$ using $\left(\varsigma_{i j}\right)_{S}$ and $\left(\varsigma_{i j}\right)_{T}$.

After setting $p=N+1-i$ and $q=N+1-j$, we can rewrite Equation (5) of Song [9] as:

$$
\delta \hat{\phi}_{u n c}(t)=\sum_{p=1}^{N-1} \sum_{q=p+1}^{N}\left(\varsigma_{N+1-p, N+1-q}\right)_{S} \Delta \alpha_{p} \times \Delta \alpha_{q}
$$

If $\delta \hat{\phi}_{\text {unc }}(t)$ and $\left(\varsigma_{N+1-p, N+1-q}\right)_{S}$ are respectively denoted by $\delta \hat{\phi}_{l}$ and $\xi_{p q}$, Equation (a1) can be rewritten as:

$$
\delta \hat{\phi}_{l}=\sum_{p=1}^{N-1} \sum_{q=p+1}^{N} \xi_{p q} \Delta \alpha_{p} \times \Delta \alpha_{q}
$$

Comparing Equation (a2) with the [1] Equation (3), we will find that both equations are the same expression under $\xi_{p q}=\left(\varsigma_{i j}\right)_{T}$ with $p=i$ and $q=j$.

Thus, to make Song [9] Equation (5) of and [1] Equation (3) equivalent will achieve $\left(\varsigma_{i j}\right)_{T}=\left(\varsigma_{N+1-i, N+1-j}\right)_{S}$. Using this relationship, we have respectively converted $\varsigma_{i j} \mathrm{~S}$ in Tables 1 and 2 of Song [9] to $\varsigma_{i j} \mathrm{~s}$ in New Tables 9 and 10, also we can convert Song [9] Equation (13) to [1] Equation (14), when Song [9] $n$ is replaced by $L$.

Now we rewrite Song [9] Equation (59) as: 


$$
\begin{aligned}
& \delta_{\phi_{u n c}}-\delta \phi_{c}=z_{3}^{\prime} \omega\left(t_{m-1}\right) \times \dot{\omega}\left(t_{m-1}\right)\left(t-t_{m-1}\right)^{3}+z_{4}^{\prime} \omega\left(t_{m-1}\right) \times \ddot{\omega}\left(t_{m-1}\right)\left(t-t_{m-1}\right)^{4} \\
&+\left(z_{51}^{\prime} \omega\left(t_{m-1}\right) \times \dddot{\omega}\left(t_{m-1}\right)+z_{52}^{\prime} \dot{\omega}\left(t_{m-1}\right) \times \ddot{\omega}\left(t_{m-1}\right)\right)\left(t-t_{m-1}\right)^{5} \\
&+\left(z_{61}^{\prime} \omega\left(t_{m-1}\right) \times \ddot{\ddot{\omega}}\left(t_{m-1}\right)+z_{62}^{\prime} \dot{\omega}\left(t_{m-1}\right) \times \ddot{\omega}\left(t_{m-1}\right)\right)\left(t-t_{m-1}\right)^{6} \\
&+\left(z_{71}^{\prime} \omega\left(t_{m-1}\right) \times \ddot{\omega}\left(t_{m-1}\right)+z_{72}^{\prime} \dot{\omega}\left(t_{m-1}\right) \times \ddot{\omega}\left(t_{m-1}\right)+z_{73}^{\prime} \ddot{\omega}\left(t_{m-1}\right) \times \dddot{\omega}\left(t_{m-1}\right)\right)\left(t-t_{m-1}\right)^{7} \\
&+\mathrm{o}\left(\left(t-t_{m-1}\right)^{9}\right) \\
& z_{3}^{\prime}=\frac{1}{6}\left(f_{3}-\frac{1}{2}\right), z_{4}^{\prime}=\frac{1}{24}\left(f_{4}-1\right), z_{51}^{\prime}=\frac{1}{120}\left(f_{51}-\frac{3}{2}\right), z_{52}^{\prime}=\frac{1}{120}\left(f_{52}-1\right), z_{61}^{\prime}=\frac{1}{720}\left(f_{61}-2\right) \\
& z_{62}^{\prime}=\frac{1}{720}\left(f_{62}-\frac{5}{2}\right), z_{71}^{\prime}=\frac{1}{5040}\left(f_{71}-\frac{5}{2}\right), z_{72}^{\prime}=\frac{1}{5040}\left(f_{72}-\frac{9}{2}\right), z_{73}^{\prime}=\frac{1}{5040}\left(f_{73}-\frac{5}{2}\right)
\end{aligned}
$$

where $f$ s are of Song [9], rather than of [1].

Set:

$$
g_{i}=\frac{d^{i-1}}{d t^{i-1}}\left(\left.\omega(t)\right|_{t=t_{m-1}}\right) /(i-1) !, i=1,2, \ldots
$$

where $i$ is a positive integer, and $\frac{d^{0}}{d t^{0}}\left(\left.\omega(t)\right|_{t=t_{m-1}}\right)$ denotes $\omega\left(t_{m-1}\right)$.

Then Equation (a3) can be converted into [1] Equation (13), when $\delta \hat{\phi}_{u n c}(t)-\delta \phi_{c}(t), t_{m-1}$ and $n$ are respectively replaced by $\delta \hat{\phi}_{l}-\delta \phi_{l}, t_{l-1}$ and $L$.

To confirm the correctness of [1] Equations (13) and (14), the $z \mathrm{~s}$ in [1] Equation (13) are calculated for LMSuc using the [1] $f \mathrm{~s}$ (see [1] Equation (14)) and $\varsigma \mathrm{s}$ in New Table 10. Also the $z^{\prime} \mathrm{s}$ in Equation (a3) are calculated for UncExp using the Song [9] $f$ s (see Song [9] Equations (13)) and $\varsigma \mathrm{s}$ in Song [9] Table 1. The $z \mathrm{~s}$ for LMSuc and the $z^{\prime} \mathrm{s}$ for UncExp are listed in Tables a1 (the copy of [1] Table 16) and a2, respectively.

Table a1. The $z$ s for [1] LMSuc.

\begin{tabular}{ccccccccccc}
\hline $\boldsymbol{L}$ & $\boldsymbol{N}$ & $\boldsymbol{z}$ & $\boldsymbol{z} 4$ & $\boldsymbol{z 5 1}$ & $\boldsymbol{z 5 2}$ & $\boldsymbol{z 6 1}$ & $\boldsymbol{z} \mathbf{6 2}$ & $\boldsymbol{z} \mathbf{7 1}$ & $\boldsymbol{z} \mathbf{7 2}$ & $\boldsymbol{z} \mathbf{7 3}$ \\
\hline 3 & 3 & $-2.29 \mathrm{e}-5$ & 0 & $-9.12 \mathrm{e}-4$ & $-2.56 \mathrm{e}-4$ & $-1.83 \mathrm{e}-3$ & $-8.46 \mathrm{e}-4$ & $-2.57 \mathrm{e}-3$ & $-1.48 \mathrm{e}-3$ & $-5.53 \mathrm{e}-4$ \\
4 & 4 & $4.95 \mathrm{e}-7$ & $-1.30 \mathrm{e}-8$ & $-2.00 \mathrm{e}-8$ & $-1.04 \mathrm{e}-6$ & $1.32 \mathrm{e}-7$ & $-1.02 \mathrm{e}-8$ & $-6.17 \mathrm{e}-5$ & $-7.30 \mathrm{e}-5$ & $-2.84 \mathrm{e}-5$ \\
5 & 5 & $1.07 \mathrm{e}-8$ & $1.07 \mathrm{e}-9$ & $2.24 \mathrm{e}-9$ & $2.21 \mathrm{e}-8$ & $3.03 \mathrm{e}-9$ & $1.55 \mathrm{e}-9$ & $-3.49 \mathrm{e}-5$ & $2.08 \mathrm{e}-9$ & $3.45 \mathrm{e}-6$ \\
\hline
\end{tabular}

Table a2. The $z^{\prime} \mathrm{s}$ for Song [9] UncExp.

\begin{tabular}{ccccccccccc}
\hline $\boldsymbol{n}$ & $\boldsymbol{N}$ & $\boldsymbol{z}^{\prime}$ & $\boldsymbol{z}^{\prime}$ & $\boldsymbol{z 5}^{\prime}$ & $\boldsymbol{z 5 5}^{\prime}$ & $\boldsymbol{z 6 1}^{\prime}$ & $\boldsymbol{z}^{\prime} \mathbf{2}^{\prime}$ & $\boldsymbol{z}_{\mathbf{7 1}}{ }^{\prime}$ & $\boldsymbol{z}_{\mathbf{2}}{ }^{\prime}$ & $\boldsymbol{z}_{\mathbf{2}}{ }^{\prime}$ \\
\hline 3 & 3 & $-2.29 \mathrm{e}-5$ & 0 & $-1.52 \mathrm{e}-4$ & $-1.28 \mathrm{e}-4$ & $-7.63 \mathrm{e}-5$ & $-1.41 \mathrm{e}-4$ & $-2.15 \mathrm{e}-5$ & $-6.18 \mathrm{e}-5$ & $-4.61 \mathrm{e}-5$ \\
4 & 4 & $4.95 \mathrm{e}-7$ & $-6.51 \mathrm{e}-9$ & $-3.34 \mathrm{e}-9$ & $-5.21 \mathrm{e}-7$ & $5.49 \mathrm{e}-9$ & $-1.70 \mathrm{e}-9$ & $-5.14 \mathrm{e}-7$ & $-3.04 \mathrm{e}-6$ & $-2.37 \mathrm{e}-6$ \\
5 & 5 & $1.07 \mathrm{e}-8$ & $5.33 \mathrm{e}-10$ & $3.73 \mathrm{e}-10$ & $1.10 \mathrm{e}-8$ & $1.26 \mathrm{e}-10$ & $2.58 \mathrm{e}-10$ & $-2.91 \mathrm{e}-7$ & $8.67 \mathrm{e}-11$ & $2.87 \mathrm{e}-7$ \\
\hline
\end{tabular}

Comparing the $z_{3}^{\prime}, z 4^{\prime}, z 51^{\prime}$ and $z 52^{\prime}$ in Table a2 with those in Equations (65)-(67) of Song [9], we can find that the former is consistent with the later except for $z 4^{\prime}$ and $z 51^{\prime}$. (The $z 4^{\prime}$ and $z 51^{\prime}$ in Equations (66) and (67) of Song [9] are zero, while the $z 4^{\prime}$ and $z 51^{\prime}$ in Table a2 for UncExp4 and UncExp5 are near 
zero. The difference between $z 4^{\prime}$ and $z 51^{\prime}$ of Table a 2 and those of Song [9] is due to round-off (to six places) in the Song [9] $\varsigma$ s used in [1].) This has been confirmed independently by a Reviewer of [1] that found identical results when using Song [9] equations and Song rounded $\varsigma s$.

The authors wish to express their appreciation to a reviewer of [1] for his insightful comments and constructive suggestions used in the original article, also for his valuable suggestions used in this correction.

\section{References}

1. Tang, C.Y.; Chen, X.Y. A Class of Coning Algorithms Based on a Half-Compressed Structure. Sensors 2014, 14, 14289-14301.

(C) 2015 by the authors; licensee MDPI, Basel, Switzerland. This article is an open access article distributed under the terms and conditions of the Creative Commons Attribution license (http://creativecommons.org/licenses/by/4.0/). 